\title{
Identification of Key Iron Metabolism-Related Long Non-Coding RNAs in Hepatocellular Carcinoma Based on Bioinformatics Analysis
}

yu chen ( $\nabla$ chenyutmr@163.com )

Tianjin Hospital of ITCWM Nankai Hospital

Research

Keywords: Hepatocellular carcinoma, Iron metabolism, LncRNA, ceRNA network

Posted Date: August 4th, 2021

DOI: https://doi.org/10.21203/rs.3.rs-754313/v1

License: (c) (i) This work is licensed under a Creative Commons Attribution 4.0 International License.

Read Full License 


\section{Abstract}

Background $\mathbb{X}$ The present study explored the regulatory mechanisms and functional roles of iron metabolism-related long non-coding RNAs (IncRNAs) in hepatocellular carcinoma (HCC) and their potential impact on prognosis of HCC patients.

Methods $₫$ RNA-seq data and clinical information of HCC samples and normal samples were downloaded from The Cancer Genome Atlas (TCGA) database and International Cancer Genome Consortium (ICGC) portal. Iron metabolism-related genes were downloaded from Reactome database and AmiGo2 database. Differential expression and correlation analysis were performed to identify iron metabolism-related differentially expressed IncRNAs (DEIncRNAs). Moreover, Kaplan-Meier (KM) survival and receiver operating characteristic (ROC) analysis were used to screen the possible prognostic and diagnostic biomarkers of HCC.

Results: A total of 20 differentially expressed and iron metabolism-related genes (DEIMRG) were identified by overlapping 3746 differentially expressed genes (DEGs) and 86 IMRGs. Next, ARHGAP11B, LINC00205, LINC00261 and SNHG12 were screened through Univariate Cox regression. Kaplan-Meier survival curves indicated that ARHGAP11B, LINC00205, LINC00261 and SNHG12 were related to overall survival (OS) in HCC patient in TCGA database. ARHGAP11B, LINC00205 and LINC00261 were finally identified as prognostic DEIMRGs related with OS of HCC patients after validate the survival results in ICGC portal. ARHGAP11B, LINC00205 and LINC00261 all achieved an AUC value of $>0.80$ in ROC curve analysis. Furthermore, LINC00205 was identified as independently prognostic factor by multivariate Cox analysis combined with clinicopathological factors. Moreover, a ceRNA network including 25 DEmRNAs, 15 DEmiRNA and 3 DElncRNAs was successfully constructed, based on prognostic DElncRNAs and key target miRNAs and mRNAs of them predicted by starBase database and miRwalk. The PPI network illustrated that CDC25A, CHEK1, CCNE2 and ANLN proteins interact more with other proteins.

Conclusions: In the present study, we identified iron metabolism related LINC00205 as a prognostic and diagnostic biomarker and constructed a metabolism-related ceRNA network, which may contribute to the treatment of HCC.

\section{Introduction}

Hepatocellular carcinoma (HCC) is still one of the most common cancer and leading cause of cancer mortality worldwide, especially in China[1, 2]. In china, the major risk causes of HCC, including chronic infection with hepatitis B virus (HBV), hepatitis $C$ virus (HCV), excess alcohol consumption, continued to drive the increasing burden of $\mathrm{HCC}$ over the past decades[3, 4]. Despite remarkable improved the 5-year survival, the rate of detection and diagnosis of HCC at early stage was relatively low in China[5] . Moreover, most of patients were diagnosed as advanced-stage HCC at the time of presentation[6]. Although effective therapeutic approaches had been achieved, the prognosis of HCC remains poor[7]. 
Therefore, identification of novel biomarkers for predicting the prognosis and improving the diagnosis of HCC patients was urgent for the personalized treatment of HCC.

Long noncoding RNAs (IncRNAs), whose lengths are more than 200 nucleotides, have been revealed to have essential roles in different biological functions and process[8, 9]. Especially, IncRNAs plays a critical role in the occurrence and development of various types of cancers $[10,11]$. Iron as one of the most and essential elements in human body, is involved in several pathological and physiological processes like including cellular respiration, DNA repair and synthesis, cell death, energy metabolism, and oxygen transport [12-14]. Researches have suggested that a few IncRNAs can regulating the development of cancers by regulating iron metabolism[15]. For example, Liu et al. suggested that fetal-lethal noncoding developmental regulatory RNA (FENDRR) as an antifibrotic long noncoding RNA can reduced pulmonary fibrosis by inhibiting fibroblast activation by reducing iron concentration, which may act as a potential therapeutic target for pulmonary fibrosis[16] . Aberrant expression of IncRNAs related with iron metabolism has been participated in regulating critical physiological functions which were linked to different types of cancer in present studies[17-19]. Moreover, a review revealed that hepatic iron accumulation can lead to fibrotic responses, and the significance and potential of iron-related proteins in the progression and development of liver fibrosis[20] . On the other hand, Miyanishi et al. demonstrated that abnormal iron metabolism leading to hepatocyte injury which may lead to accelerate the incidence rate of hepatic inflammation[15] . However, the role of iron metabolism-related IncRNAs in HCC was still unclear.

In this study, we employed the bioinformatics approach to identify the differentially expressed genes between HCC and normal tissues from The Cancer Genome Atlas (TCGA) database and International Cancer Genome Consortium (ICGC) portal. Two original microarray datasets, from Reactome database and AmiGo2 database were also downloaded for overlapping iron metabolism-related genes. Additionally, we filtered DEIncRNAs as prognostic biomarkers for HCC using Kaplan-Meier (KM) survival and receiver operating characteristic (ROC) analysis. An iron metabolism-related ceRNA network was also constructed. The aim of this study will screen underlying survival biomarkers and discuss the potential molecular mechanism of iron metabolism related with HCC.

\section{Methods}

\section{Data acquisition}

The RNA-seq data (3 levels) and clinic information of 369 HCC tissue samples with the following-up data and 50 paracancerous tissue samples were downloaded from TCGA (https://portal.gdc.cancer.gov/) database. At the same time, RNA-seq data and clinical information of another 231 tumor samples and 202 normal samples were obtained from the International Cancer Genome Consortium (ICGC) portal (https://dcc.icgc.org/projects/LIRI-JP). Besides, the iron metabolism-related genes were downloaded from Reactome database (https://reactome.org) and AmiGo2 database (http://amigo.geneontology.org) using the following search terms were: "Homo sapiens," and "cellular iron ion homeostasis". After careful 
screening by overlapping iron metabolism-related genes (IMRGs) from the Reactome database and the AmiGo2 database, 85 IMRGs were selected for subsequent analysis.

\section{Differential expression analysis}

The "edgeR" package was utilized to identifying differentially expressed genes (DEGs), miRNA (DEmiRNAs), and IncRNAs (DElncRNAs) between HCC tissue samples and paracancerous tissue samples in the TCGA cohort [21]. The thresholds were set as $\mid$ log2 foldchange (FC) $\mid>1.0$ and $P<0.05$. Differentially expressed iron metabolism-related genes (DEIMRGs) were identified by overlapping DEGs and IMRGs. In addition, volcano plots and heatmap were drawn respectively by "ggplot2 3.3.2" and "pheatmap 0.7.7" package in R were used to show the expression of DEIMRGs and DElncRNAs.

\section{Functional enrichment analysis}

To explore the functions related to biological process (BP), cellular component (CC) and molecular function (MF) of DEIMRGs, GO (Gene Ontology) function and KEGG (Kyoto Encyclopedia of Genes and Genomes) pathway enrichment analyses of the DEIMRGs were performed through "clusterProfiler" package in $\mathrm{R}[22]$. Adjust $\mathrm{P}<0.05$ were set as the cutoff criteria.

\section{Correlation analysis}

Based on the purpose of investigating the relationship of DEIMRGs and DElncRNAs, pearson correlation analysis was performed by "cor.test()" function and the result of correlation analysis was visualized with heatmap drawed by the "ggplot2" package. DElncRNAs with correlation coefficient more than 0.4 and $\mathrm{P}$ value lower than 0.05 were selected and defined as iron metabolism-related DEIncRNAs for subsequent analysis.

\section{Survival Analysis}

Cox proportional-hazards regression model was a standard method for modelling censored life-time data with covariates, having achieved widespread be used in the analysis of time-to-event data[23]. Hence, univariate Cox regression analysis was performed to screen possible prognostic DElncRNAs from the iron metabolism-related DElncRNAs by the "survival" package in the TCGA cohort[24] with a $P$-value $<0.05$. Then, Kaplan-Meier (KM) plots were applied to assess the differences in survival between the high- and low- expression groups based on the median expression value of each IncRNA. In addition, we also further validated the expression levels in HCC vs. non-tumor liver tissues of the possible prognostic DElncRNAs using log-rank tests and employed survival analysis of these possible prognostic DElncRNAs in the ICGC database.

\section{Identification of the diagnostic biomarkers}

Receiver operating characteristic (ROC) analysis was carried out to evaluate the sensitivity and specificity of the prognostic DElncRNAs for HCC diagnosis using the 'pROC' R package[25]. An area under the curve (AUC) value was calculated and used to test the diagnostic effectiveness. 


\section{The clinical significance of the possible prognostic and diagnostic biomarkers}

To explore the clinical significance of the prognostic and diagnostic DElncRNAs, we further compared the expression levels of prognostic and diagnostic DEIncRNAs in different clinical features of the TCGA cohort through log-rank tests. A value of $\mathrm{P}<0.05$ was considered to indicate a statistically significant difference.

\section{Independent prognostic analysis}

In order to further investigate whether prognostic and diagnostic DElncRNAs could be used as independent prognostic factors, univariate Cox regression analysis integrating prognostic and diagnostic DElncRNAs and clinic pathological parameters was firstly carried out to screen independent prognostic factors in the TCGA cohort, with the screening condition was $P$-value $<0.05$. Subsequently, multivariate Cox regression analysis further was performed to further screening. Finally, the results of univariate and multivariate Cox analysis were shown by forest maps.

\section{Construction of competing endogenous (ce)RNA regulatory network}

To further explore the regulation mechanism of prognostic and diagnostic DElncRNAs, StarBase datebase (http://starbase.sysu.edu.cn/) and miRwalk was used to predict the targeting miRNAs and mRNA. Next, by overlapping predictive miRNA and DEmiRNAs which were oppositely expressed compared to IncRNAs, and predictive mRNA and DEmRNAs which were oppositely expressed compared to miRNAs, key miRNAs and mRNNAs were selected for constructing a ceRNA network. Finally, a ceRNA regulatory network was constructed by integrating the key IncRNA-miRNA and miRNA-mRNA regulatory relationships via Cytoscape[26]. Besides, the genes in the constructed ceRNA regulatory network were used for $\mathrm{GO}$ and KEGG function analysis to identify the important biological process and signaling pathways. Ultimately, a protein-protein interaction networks (PPI) was constructed to show the interaction of these genes with the help of STRING (https://string-db.org/) and Cytoscape[27].

\section{Statistical analysis}

Univariate and multivariate Cox regressions analyses were conducted by using the "survival" package in $\mathrm{R}$. The OS of the high- and low-expression subgroups were compared using the Kaplan-Meier method with a log-rank test. A p-value of less than 0.05 was considered to be statistically significant.

\section{Results}

\section{Differential Expression Analysis}

A total of 3746 DEGs (1064 down-regulated and 2682 upregulated genes) and 135 DElncRNA (10 downregulated and 125 upregulated mRNAs) were identified between HCC tissue samples and normal 
tissue samples in the TCGA cohort (Figs. 1A and 1B).

In addition, 20 DEIMRGs including 11 down-regulated and 9 upregulated IMRGs, were identified by overlapping DEGs and IMRGs from the Reactome database and the AmiGo2 database. The expression levels of 20 DEIMRGs were shown by volcano plot and heatmap (Figs. 1C and 1D).

\section{GO an KEGG functional enrichment analysis of DEIMRGs}

In order to explore the biological function of DEIMRGs, GO functional annotation and KEGG pathway enrichment analysis were performed by "clusterProfiler" package in R. As shown in the (Fig. 2A), the GO biological process analysis suggested that most of these genes were mainly associated with iron ion transport, cellular transition metal ion homeostasis and cellular transition metal ion homeostasis. Moreover, these genes were mainly related to the vacuolar proton-transporting V-type ATPase complex, proton-transporting V-type ATPase complex and proton-transporting two-sector ATPase complex as revealed by $\mathrm{GO}$ cellular component analysis. $\mathrm{GO}$ molecular function analysis showed these genes were mainly involved in proton-exporting ATPase activity, phosphorylative mechanism, ATPase activity, coupled to transmembrane movement of ions, rotational and mechanism, and proton-transporting ATPase activity, rotational mechanism. Furthermore, KEGG pathway analysis of DEIMRGs revealed that these DEIMRGs were mainly involved in ferroptosis, synaptic vesicle cycle and oxidative phosphorylation etc pathways (Fig. 2B). In a word, these results suggested that iron metabolism-related genes play a key role in the occurrence of HCC.

\section{Identification of the iron metabolism-related DElncRNAs}

To screen iron metabolism-related DElncRNAs from DElncRNAs, pearson correlation analysis was performed by "cor.test)" function. The results showed that TFR2, HPX and TF were significantly negatively correlated with most DElncRNAs, while ATP6V1C2, ATP6V1C1, FLVCR1 and ATP6V1B1 were positively correlated with most DEIncRNAs (Fig. 3). In addition, a total of 33 iron metabolism-related DEIncRNAs were identified for subsequent analyses based on their high correlation with the DEIMRGs (|R| $>0.4, p<0.05)$.

\section{Survival Analysis}

To further assess the prognostic significance of 33 iron metabolism-related DElncRNAs, univariate Cox regression analysis was used to evaluate the associations between the expression levels of 33 iron metabolism-related DEIncRNAs and the OS of HCC patients from the TCGA cohort. The results of univariate Cox regression analysis showed that four iron metabolism-related DElncRNAs including ARHGAP11B, LINC00205, LINC00261 and SNHG12 were related to the OS of HCC patients (Table 1). 
Table 1

univariate Cox regression analysis showed that four iron metabolism-related DElncRNAs

\begin{tabular}{|lllll|}
\hline Gene & HR & HR.95L & HR.95H & coxPvalue \\
\hline ARHGAP11B & 2.408171 & 1.275171 & 4.547851 & 0.006742 \\
\hline LINC00261 & 0.985387 & 0.972071 & 0.998885 & 0.033954 \\
\hline LINC00205 & 1.229129 & 1.095746 & 1.37875 & 0.000431 \\
\hline SNHG12 & 1.132282 & 1.034761 & 1.238993 & 0.00686 \\
\hline
\end{tabular}

Table 1. univariate Cox regression analysis showed that four iron metabolism-related DElncRNAs

Interesting, the Kaplan-Meier survival curves also showed that ARHGAP11B, LINC00205, LINC00261 and SNHG12 were associated with the OS of HCC patients. Obviously, higher expressions of ARHGAP11B (HR 1.43 [1.01-2.03], $P=0.04$ ), LINC00205 (HR 1.44 [1.02-2.04], $P=0.036)$, SNHG12 (HR 1.63 [1.15-2.3], $P=$ 0.005]) were related to the worse OS in HCC patients (Figs. 4A-C), while a low expression of LINC00261 (HR 0.61 [0.43-0.86], $\mathrm{P}=0.005$ ) was associated with better outcome in HCC (Fig. 4D). The red lines represented the high expression of iron metabolism-related DElncRNAs in HCC, and the green lines represented low expression of iron metabolism-related DElncRNAs.

Notably, consistent with the results of TCGA cohort, the results of survival and expression analysis using ICGC data (HR 2.13 [1.16-3.9], $\mathrm{P}=0.017$ ) also suggested that ARHGAP11B also was differentially expressed and related to the OS of HCC patients (Figs. 4E and 4F). However, the SNHG12 was not associated with the OS of HCC patients (HR 1.49 [0.81-2.74], $\mathrm{P}=0.194$ ) (Fig. 4G). Moreover, as for the resting two IncRNAs, we could not find them in the expression matrix of ICGC database. Therefore, ARHGAP11B, LINC00205, LINC00261 were selected as the prognostic biomarkers.

\section{Identification of diagnostic biomarkers}

To further explored the diagnostic value of the prognostic biomarkers, we performed the ROC curve analysis in the TCGA cohort to evaluate their sensitivity and specificity for the diagnosis of HCC. As shown in the (Fig. 5A-C), all of the three prognostic biomarkers (ARHGAP11B, LINC00205 and LINC00261) achieved an AUC value of $>0.80$, demonstrating that these DElncRNAs have high sensitivity and specificity for HCC diagnosis. Hence, these results suggested that ARHGAP11B, LINC00205 and LINC00261 could be used as biomarkers for the diagnosis of HCC.

\section{The clinical significance of the possible prognostic DElncRNAs}

To confirm the clinical value of the prognostic biomarkers, firstly, we compared the expression levels of these three DElncRNAs in different sugroup stratified by molecular subtypes, age (over or under 60 years), sex (male/female), pathological stage (stage III, IV/stage I, II), T stage (stage III, IV/stage I, II), N stage 
(presence of lymphatic metastasis or not) and $M$ stage (presence of distant metastasis or not) in the TCGA database, separately. As shown in the (Fig. 6), the expression of ARHGAP11B was significantly different in patients with different clinical features such as age, gender, $T$ stage and pathological stage, while the expression levels of LINC00205 were associated with gender and pathological stage. Clearly, the patients under 60 years old, females, stage with III, IV and T stage with III, IV inclined to have higher expression level of ARHGAP11B compared with those patients with the older age, males, stage with I, II and T stage with I, II. In addition. the expression level of LINC00205 was higher in females than males. Besides, the expression levels of LINC00205 in stage III, IV subgroup were higher compare with stage I, II subgroup.

\section{Independent prognostic analysis}

Integrating the possible prognostic DElncRNAs and clinic pathological parameters, three DElncRNAs including ARHGAP11B, LINC00205, stage and T stage were identified to be associated with prognosis by univariate Cox regression analysis (Fig. 7A). Finally, LINC00205 was independently associated with the prognosis (Fig. 7B).

\section{ceRNA regulatory network construction and analysis}

To explore the regulatory mechanism of the diagnostic and prognostic DElncRNAs, we firstly screened 91 predictive miRNAs regulated by ARHGAP11B. Then, after overlapping down-regulating DEmiRNAs (because of the up-regulating ARHGAP11B in cancer samples, Supplementary Fig. 1), 3 key downregulated miRNAs which might be regulated by ARHGAP11B were selected. Subsequently, 13 key mRNA which might be regulated by 3 key down-regulated miRNAs were identified through the analyses of the expression, starBase database and miRwalk (Fig. 8A). Similarly, we also identified the target key miRNAs and mRNAs of LINC00205 and LINC00261. Finally, the ceRNA regulatory network including 3 IncRNAs, 15 miRNAs and 25 mRNAs was constructed as shown (Fig. 8A). In addition, GO and KEGG pathway function analysis was performed to assess the biological process of the genes in the constructed ceRNA regulatory network and the results illustrated that steroid hormone mediated signaling pathway, steroid hormone receptor activity and nuclear division etc biological processes and cell cycle, cellular senescence and p53 signaling pathway etc signaling pathways were significant enriched (Figs. 8B and 8C). Ultimately, a protein-protein interaction networks (PPI) was constructed to show the interaction of these genes. As shown(Fig. 8D), CDC25A, CHEK1, CCNE2 and ANLN proteins interact more with other proteins.

\section{Discussion}

The homeostasis of iron metabolism is crucial in biological systems. Alterations in iron metabolism is associated with a number of pathologies to hepatic carcinogenesis that can be ultimately fatal[28]. Evidence continues to accumulate that disorder of iron metabolism in the hepatocytes is closely associated with progression and development of hepatic inflammation as well as hepatocellular carcinoma [29]. There is a positive correlation between the risk of HCC and increased iron level in hepatocytes [30]. 
Furthermore, several researches suggest that gene-IncRNA module involved in iron metabolism is an important risk factor for the pathogenesis and progression of different diseases. Up till now, few IncRNAs related with iron metabolism have been identified in $\mathrm{HCC}[31]$.

In my present study, we firstly identify 3 iron metabolism-related IncRNAs as the prognostic and diagnostic biomarkers of HCC. Long intergenic non-protein coding RNA 205 (LINC00205), which is located at chromosome 21, NC000021.9, has been revealed to act as a novel biomarker for pancreatic cancer and lung cancer[32]. In our study, we further reveal that LINC00205 may be associated with the occurrence of HCC and affect the prognosis of HCC. Consistent with our results, LINC00205 can inhibit the proliferation and migration of liver cancer cells, which may finally lead to a better prognosis by modulating the miR-184/EPHX1 pathway in HCC patients[33]. It has been reported that overexpression of LINC00261 can inhibit cell proliferation, cell colony formation, cell invasion and EMT process in HCC cells by activating the DNA damage response[34] [35]. Marta Florio et al [36]. demonstrate ARHGAP11B amplifies basal progenitors and is capable of causing neocortex folding in mouse.

Moreover, we construct an iron metabolism-related ceRNA network. RACGAP1 as a hub gene may be involved in the carcinogenesis, invasion or recurrence of HCC, which promotes proliferation of HCC cells by reducing activation of the Hippo and YAP pathways [37]. Zhang et al. identify several novel mutated genes including GNAL may play key roles in liver tumorigenesis[38] .

The PPI network of target genes in the ceRNA network suggest that CDC25A, CHEK1, CCNE2 and ANLN proteins interact more with other proteins. As an oncogene, higher expression of CDC25A is detected in various types of cancer, which is significantly associated with progress of tumor differentiation and invasion $[39,40]$. The inhibition of CHEK1 may result breast cancer cell apoptosis and enhancing replication stress in breast cancer cells expressing RNF126[41]. Overexpression of CHEK1 may induce HCC progression in vitro and accelerate tumor formation in nude mice,which is identified as a target gene of miR-330-5p[42].The key findings of one study demonstrate that positively regulation of the expression of CCNE2 as the target of miR-577 via circ-CSPP1 may promoted HCC cell colony formation, invasion and migration in vitro[43] .

Several important KEGG pathways are identified,among them the most significantly activated pathways are cell cycle, cellular senescence and p53 signaling pathway. A study demonstrated that Cyclin D1, a protein involved in G1-phase of cell cycle, are overexpressed in the iron-overloaded liver[44]. In human hepatoma SMMC7721 cells $₫$ the reduction of the cellular iron content induces alterations of p53-p27-p21 signaling to arrest the cell cycle at $S$ phase[45].In mouse ageing hepatic tissue, researchers reveal that iron storage levels provided a robust biomarker of cellular senescence, for associated iron accumulation and for resistance to iron-induced toxicity [46]. p53 regulated iron metabolism through the transcriptional regulation of ISCU (iron-sulfur cluster assembly enzyme), which may play a key role in the maintenance of iron homeostasis in hepatocellular carcinogenesis[47] .

Go enrichment analysis is performed to demonstrated that hub genes enriched in steroid hormone receptor binding and steroid hormone receptor activity illustrated ceRNA regulation network played an 
essential role in iron metabolism[48, 49]. Excess intracellular iron can damage DNA via reactive oxygen species (ROS) accumulation when the toxicity of ROS is at a sublethal level, resulting in cellular senescence as well as cell death[50,51]. Cancer is linked to cytotoxicity due to iron-mediated ROS, ischaemia-reperfusion injury during transplantation and ageing-related neurodegenerative diseases[52] .In mice model,Zhu et al. reveal that knockdown of ANLN in hepatocytes may blocks cytokinesis promote tumorigenesis and inhibits development of liver tumors[53] .

\section{Conclusions}

However, our study still has several limitations. First, although independently prognostic related IncRNA involved in iron metabolism was identified and validated in various independent datasets, further studies should be conducted to provided new insight into the mechanisms of HCC. Moreover, the ceRNA network was identified in three online datasets, further experimental study was urgent to verify the results of the present study. Moreover, all the results are based on bioinformatics models, more clinical cases are critical to verify the findings. Future experiments of the role of iron metabolism in the progression of HCC also needs to be further studied and discussed.

In the present study, we identify iron metabolism related LINC00205 acted as a prognostic biomarker and constructed a mRNA-miRNA-IncRNA ceRNA network. It offers potential therapeutic targets and new challenges to progression of liver tumor pathogenesis and invasion related with iron metabolism.

\section{Abbreviations}

IncRNAs, long non-coding RNAs; HCC, hepatocellular carcinoma;TCGA, The Cancer Genome Atlas; ICGC, International Cancer Genome Consortium (ICGC); ROC, receiver operating characteristic; ARHGAP11B, Rho GTPase activating protein 11B; LINC00205,long intergenic non-protein coding RNA 205; SNHG12, small nucleolar RNA host gene 12; LINC00261, long intergenic non-protein coding RNA 261; CDC25A, cell division cycle 25A; CHEK1, checkpoint kinase 1; CCNE2, cyclin E2; ANLN, anillin actin binding protein; ATP6V1C2, ATPase $\mathrm{H}+$ transporting V1 subunit C2; ATP6V1C1, ATPase $\mathrm{H}+$ transporting V1 subunit C1;FLVCR1, FLVCR heme transporter 1;ATP6V1B1, ATPase H + transporting V1 subunit B1;

\section{Declarations}

\section{Funding}

The authors received no funding for this work.

\section{Competing Interests}

The authors declare there are no competing interests.

\section{Author Contributions}

Yu Chen designed study and analyzed data. 
Yu Chen wrote and final approve the manuscript.

Availability of data and materials

The datasets supporting the conclusion of this article are included within the article.

Ethics approval and consent to participate

Not applicable

Consent for publication

Not applicable

\section{References}

1. Clark T, Maximin S, Meier J, Pokharel S, Bhargava P: Hepatocellular Carcinoma: Review of Epidemiology, Screening, Imaging Diagnosis, Response Assessment, and Treatment. Curr Probl Diagn Radiol 2015, 44:479-486.

2. Liu X, Li M, Wang X, Dang Z, Yu L, Wang X, Jiang Y, Yang Z: Effects of adjuvant traditional Chinese medicine therapy on long-term survival in patients with hepatocellular carcinoma. Phytomedicine 2019, 62:152930.

3. McGlynn KA, Petrick JL, London WT: Global epidemiology of hepatocellular carcinoma: an emphasis on demographic and regional variability. Clin Liver Dis 2015, 19:223-238.

4. Tang A, Hallouch O, Chernyak V, Kamaya A, Sirlin CB: Epidemiology of hepatocellular carcinoma: target population for surveillance and diagnosis. Abdom Radiol (NY) 2018, 43:13-25.

5. Xu XF, Xing H, Han J, Li ZL, Lau WY, Zhou YH, Gu WM, Wang H, Chen TH, Zeng YY, et al: Risk Factors, Patterns, and Outcomes of Late Recurrence After Liver Resection for Hepatocellular Carcinoma: A Multicenter Study From China. JAMA Surg 2019, 154:209-217.

6. Younossi ZM, Koenig AB, Abdelatif D, Fazel Y, Henry L, Wymer M: Global epidemiology of nonalcoholic fatty liver disease-Meta-analytic assessment of prevalence, incidence, and outcomes. Hepatology 2016, 64:73-84.

7. Khanna R, Verma SK: Pediatric hepatocellular carcinoma. World J Gastroentero/ 2018, 24:39803999.

8. Hung T, Chang HY: Long noncoding RNA in genome regulation: prospects and mechanisms. RNA Biol 2010, 7:582-585. 
9. Wilusz JE, Sunwoo H, Spector DL: Long noncoding RNAs: functional surprises from the RNA world. Genes Dev 2009, 23:1494-1504.

10. Quagliata L, Matter MS, Piscuoglio S, Arabi L, Ruiz C, Procino A, Kovac M, Moretti F, Makowska Z, Boldanova T, et al: Long noncoding RNA HOTTIP/HOXA13 expression is associated with disease progression and predicts outcome in hepatocellular carcinoma patients. Hepatology 2014, 59:911-923.

11. Bhan A, Soleimani M, Mandal SS: Long Noncoding RNA and Cancer: A New Paradigm. Cancer Res 2017, 77:3965-3981.

12. Veatch JR, McMurray MA, Nelson ZW, Gottschling DE: Mitochondrial dysfunction leads to nuclear genome instability via an iron-sulfur cluster defect. Cell 2009, 137:1247-1258.

13. Puig S, Ramos-Alonso L, Romero AM, Martínez-Pastor MT: The elemental role of iron in DNA synthesis and repair. Metallomics 2017, 9:1483-1500.

14. Oexle H, Gnaiger $\mathrm{E}$, Weiss $\mathrm{G}$ : Iron-dependent changes in cellular energy metabolism: influence on citric acid cycle and oxidative phosphorylation. Biochim Biophys Acta 1999, 1413:99-107.

15. Miyanishi K, Tanaka S, Sakamoto $\mathrm{H}$, Kato $\mathrm{J}$ : The role of iron in hepatic inflammation and hepatocellular carcinoma. Free Radic Biol Med 2019, 133:200-205.

16. Huang C, Liang Y, Zeng X, Yang X, Xu D, Gou X, Sathiaseelan R, Senavirathna LK, Wang P, Liu L: Long Noncoding RNA FENDRR Exhibits Antifibrotic Activity in Pulmonary Fibrosis. Am J Respir Cell Mol Biol 2020, 62:440-453.

17. Fan X, Liu L, Shi Y, Guo F, Wang H, Zhao X, Zhong D, Li G: Integrated analysis of RNA-binding proteins in human colorectal cancer. World J Surg Oncol 2020, 18:222.

18. Zhou T, Gao Y: Expression of Concern to: Increased expression of LncRNA BANCR and its prognostic significance in human hepatocellular carcinoma. World J Surg Onco/2019, 17:103.

19. Chen Z, Wang X, Wang G, Xiao B, Ma Z, Huo H, Li W: A seven-IncRNA signature for predicting Ewing's sarcoma. PeerJ 2021, 9:e11599.

20. Mehta KJ, Farnaud SJ, Sharp PA: Iron and liver fibrosis: Mechanistic and clinical aspects. World J Gastroenterol 2019, 25:521-538.

21. Robinson MD, McCarthy DJ, Smyth GK: edgeR: a Bioconductor package for differential expression analysis of digital gene expression data. Bioinformatics 2010, 26:139-140.

22. Yu G, Wang LG, Han Y, He QY: clusterProfiler: an R package for comparing biological themes among gene clusters. Omics 2012, 16:284-287. 
23. Fisher LD, Lin DY: Time-dependent covariates in the Cox proportional-hazards regression model. Annu Rev Public Health 1999, 20:145-157.

24. Rizvi AA, Karaesmen E, Morgan M, Preus L, Wang J, Sovic M, Hahn T, Sucheston-Campbell LE: gwasurvivr: an R package for genome-wide survival analysis. Bioinformatics 2019, 35:1968-1970.

25. Robin X, Turck N, Hainard A, Tiberti N, Lisacek F, Sanchez JC, Müller M: pROC: an open-source package for $\mathrm{R}$ and $\mathrm{S}+$ to analyze and compare ROC curves. BMC Bioinformatics 2011, 12:77.

26. Shannon P, Markiel A, Ozier O, Baliga NS, Wang JT, Ramage D, Amin N, Schwikowski B, Ideker T: Cytoscape: a software environment for integrated models of biomolecular interaction networks. Genome Res 2003, 13:2498-2504.

27. Doncheva NT, Morris JH, Gorodkin J, Jensen LJ: Cytoscape StringApp: Network Analysis and Visualization of Proteomics Data. J Proteome Res 2019, 18:623-632.

28. Tirnitz-Parker JE, Glanfield A, Olynyk JK, Ramm GA: Iron and hepatic carcinogenesis. Crit Rev Oncog 2013, 18:391-407.

29. Li Y, Xu W, Chen P, Liao M, Qin W, Liao W, Huang Z: Correlation Analysis Between Preoperative Serum Iron Level and Prognosis as Well as Recurrence of HCC After Radical Resection. Cancer Manag Res 2020, 12:31-41.

30. Rostoker G, Vaziri ND: Impact of iatrogenic iron overload on the course of hepatitis $C$ in the dialysis population: A plea for caution. Hemodial Int 2017, 21 Suppl 1:S68-s77.

31. Kontoghiorghes GJ, Kontoghiorghe CN: Iron and Chelation in Biochemistry and Medicine: New Approaches to Controlling Iron Metabolism and Treating Related Diseases. Cells 2020, 9.

32. Xie P, Guo Y: LINC00205 promotes malignancy in lung cancer by recruiting FUS and stabilizing CSDE1. Biosci Rep 2020, 40.

33. Long X, Li Q, Zhi LJ, Li JM, Wang ZY: LINC00205 modulates the expression of EPHX1 through the inhibition of miR-184 in hepatocellular carcinoma as a ceRNA. J Cell Physiol 2020, 235:3013-3021.

34. Shahabi S, Kumaran V, Castillo J, Cong Z, Nandagopal G, Mullen DJ, Alvarado A, Correa MR, Saizan A, Goel R, et al: LINC00261 Is an Epigenetically Regulated Tumor Suppressor Essential for Activation of the DNA Damage Response. Cancer Res 2019, 79:3050-3062.

35. Zhang HF, Li W, Han YD: LINC00261 suppresses cell proliferation, invasion and Notch signaling pathway in hepatocellular carcinoma. Cancer Biomark 2018, 21:575-582.

36. Florio M, Albert M, Taverna E, Namba T, Brandl H, Lewitus E, Haffner C, Sykes A, Wong FK, Peters J, et al: Human-specific gene ARHGAP11B promotes basal progenitor amplification and neocortex 
expansion. Science 2015, 347:1465-1470.

37. Li L, Lei Q, Zhang S, Kong L, Qin B: Screening and identification of key biomarkers in hepatocellular carcinoma: Evidence from bioinformatic analysis. Oncol Rep 2017, 38:2607-2618.

38. Zhang Y, Qiu Z, Wei L, Tang R, Lian B, Zhao Y, He X, Xie L: Integrated analysis of mutation data from various sources identifies key genes and signaling pathways in hepatocellular carcinoma. PLOS One 2014, 9:e100854.

39. Qin H, Liu W: MicroRNA-99a-5p suppresses breast cancer progression and cell-cycle pathway through downregulating CDC25A. J Cell Physio/ 2019, 234:3526-3537.

40. Boutros R, Lobjois V, Ducommun B: CDC25 phosphatases in cancer cells: key players? Good targets? Nat Rev Cancer 2007, 7:495-507.

41. Yang X, Pan Y, Qiu Z, Du Z, Zhang Y, Fa P, Gorityala S, Ma S, Li S, Chen C, et al: RNF126 as a Biomarker of a Poor Prognosis in Invasive Breast Cancer and CHEK1 Inhibitor Efficacy in Breast Cancer Cells. Clin Cancer Res 2018, 24:1629-1643.

42. Gong D, Feng PC, Ke XF, Kuang HL, Pan LL, Ye Q, Wu JB: Silencing Long Non-coding RNA LINC01224 Inhibits Hepatocellular Carcinoma Progression via MicroRNA-330-5p-Induced Inhibition of CHEK1. Mol Ther Nucleic Acids 2020, 19:482-497.

43. Sun Q, Yu R, Wang C, Yao J, Zhang L: Circular RNA circ-CSPP1 regulates CCNE2 to facilitate hepatocellular carcinoma cell growth via sponging miR-577. Cancer Cell Int 2020, 20:202.

44. Troadec MB, Courselaud B, Détivaud L, Haziza-Pigeon C, Leroyer P, Brissot P, Loréal O: Iron overload promotes Cyclin D1 expression and alters cell cycle in mouse hepatocytes. J Hepato/ 2006, 44:391-399.

45. Sun P, Wang S, Wang J, Sun J, Peng M, Shi P: The involvement of iron in chemerin induced cell cycle arrest in human hepatic carcinoma SMMC7721 cells. Metallomics 2018, 10:838-845.

46. Masaldan S, Clatworthy SAS, Gamell C, Meggyesy PM, Rigopoulos AT, Haupt S, Haupt Y, Denoyer $D$, Adlard PA, Bush Al, Cater MA: Iron accumulation in senescent cells is coupled with impaired ferritinophagy and inhibition of ferroptosis. Redox Biol 2018, 14:100-115.

47. Funauchi Y, Tanikawa C, Yi Lo PH, Mori J, Daigo Y, Takano A, Miyagi Y, Okawa A, Nakamura Y, Matsuda K: Regulation of iron homeostasis by the p53-ISCU pathway. Sci Rep 2015, 5:16497.

48. Feng $\mathrm{Y}$, Feng $\mathrm{Q}, \mathrm{Lv} \mathrm{Y}$, Song $\mathrm{X}, \mathrm{Qu} \mathrm{H}$, Chen $\mathrm{Y}$ : The relationship between iron metabolism, stress hormones, and insulin resistance in gestational diabetes mellitus. Nutr Diabetes 2020, 10:17. 
49. Singh AP, Fridman Y, Holland N, Ackerman-Lavert M, Zananiri R, Jaillais Y, Henn A, SavaldiGoldstein S: Interdependent Nutrient Availability and Steroid Hormone Signals Facilitate Root Growth Plasticity. Dev Cel/ 2018, 46:59-72.e54.

50. López-Otín C, Blasco MA, Partridge L, Serrano M, Kroemer G: The hallmarks of aging. Cell 2013, 153:1194-1217.

51. Sfera A, Bullock K, Price A, Inderias L, Osorio C: Ferrosenescence: The iron age of neurodegeneration? Mech Ageing Dev 2018, 174:63-75.

52. Nakamura T, Naguro I, Ichijo H: Iron homeostasis and iron-regulated ROS in cell death, senescence and human diseases. Biochim Biophys Acta Gen Subj 2019, 1863:1398-1409.

53. Zhang S, Nguyen LH, Zhou K, Tu HC, Sehgal A, Nassour I, Li L, Gopal P, Goodman J, Singal AG, et al: Knockdown of Anillin Actin Binding Protein Blocks Cytokinesis in Hepatocytes and Reduces Liver Tumor Development in Mice Without Affecting Regeneration. Gastroenterology 2018, 154:1421-1434.

\section{Figures}


$1 \mathrm{~A}$
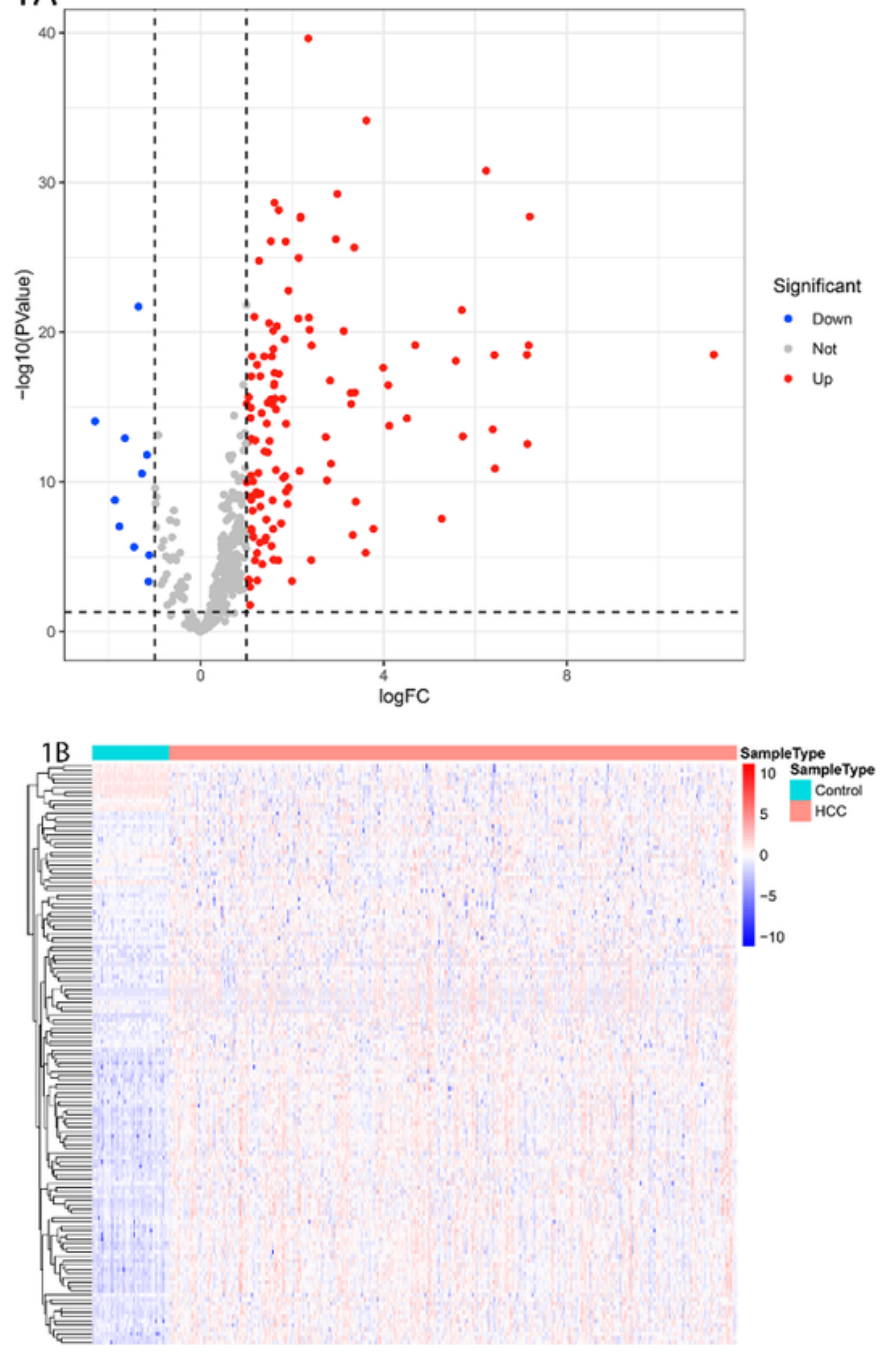

$1 C$

Significant

- Down

- Not

(
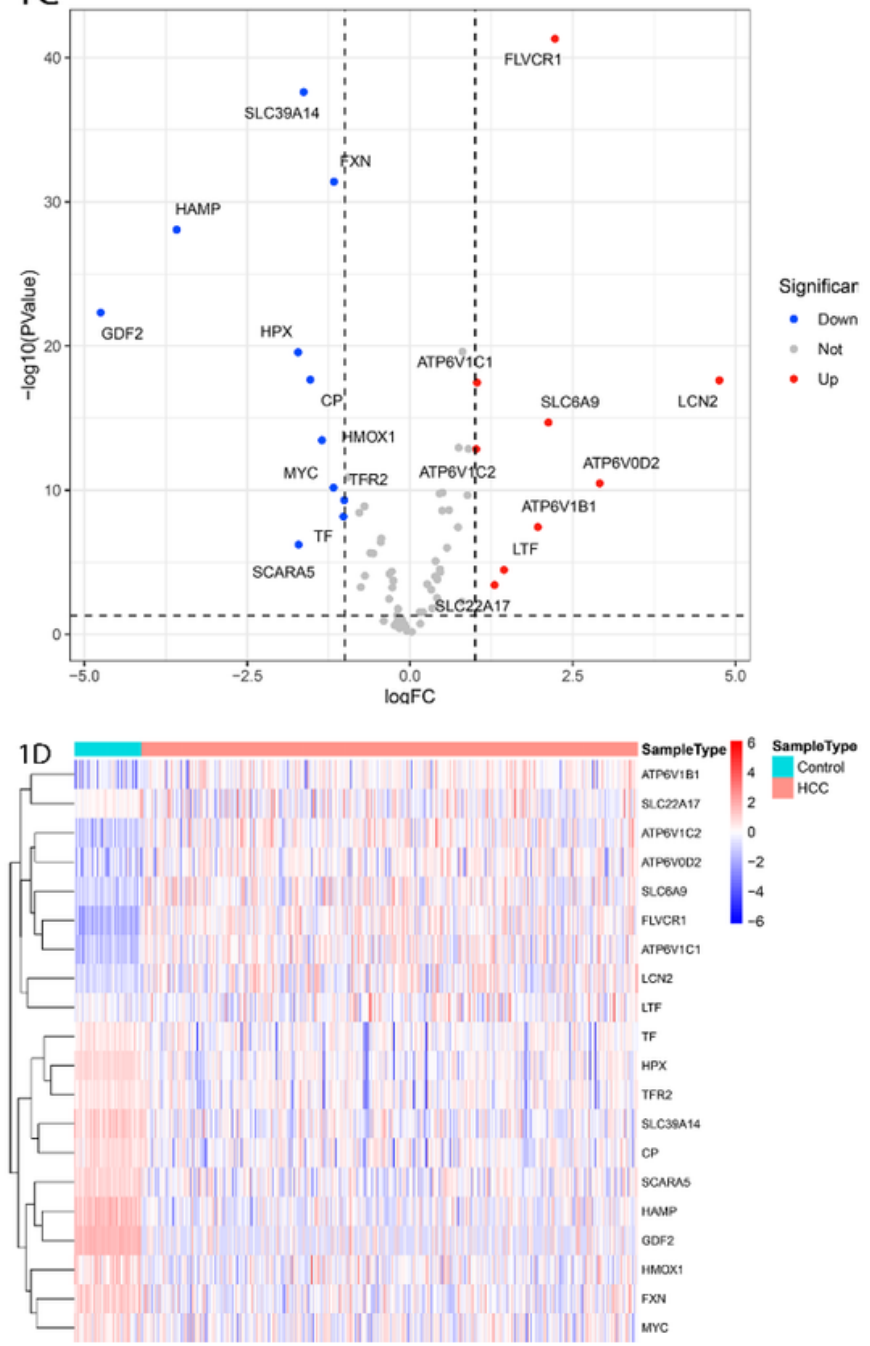

\section{Figure 1}

Differential expression analysis The red dots represented the upregulation of IncRNAs (log2FC $>1$ and adjusted $P<0: 05)$. The blue dots represented the downregulation of IncRNAs (log2FC $<-1$ and adjusted $P$ $<0: 05) A \square A$ volcano plot of DElncRNAs identified between HCC tissue samples and normal tissue samples in the TCGA cohort B) Heat map of DElncRNAs identified between HCC tissue samples and normal tissue samples in the TCGA cohort C) A volcano plot of the expression levels of 20 DEIMRGs in HCC patients D) The expression levels of 20 DEIMRGs shown heatmap in HCC patients 

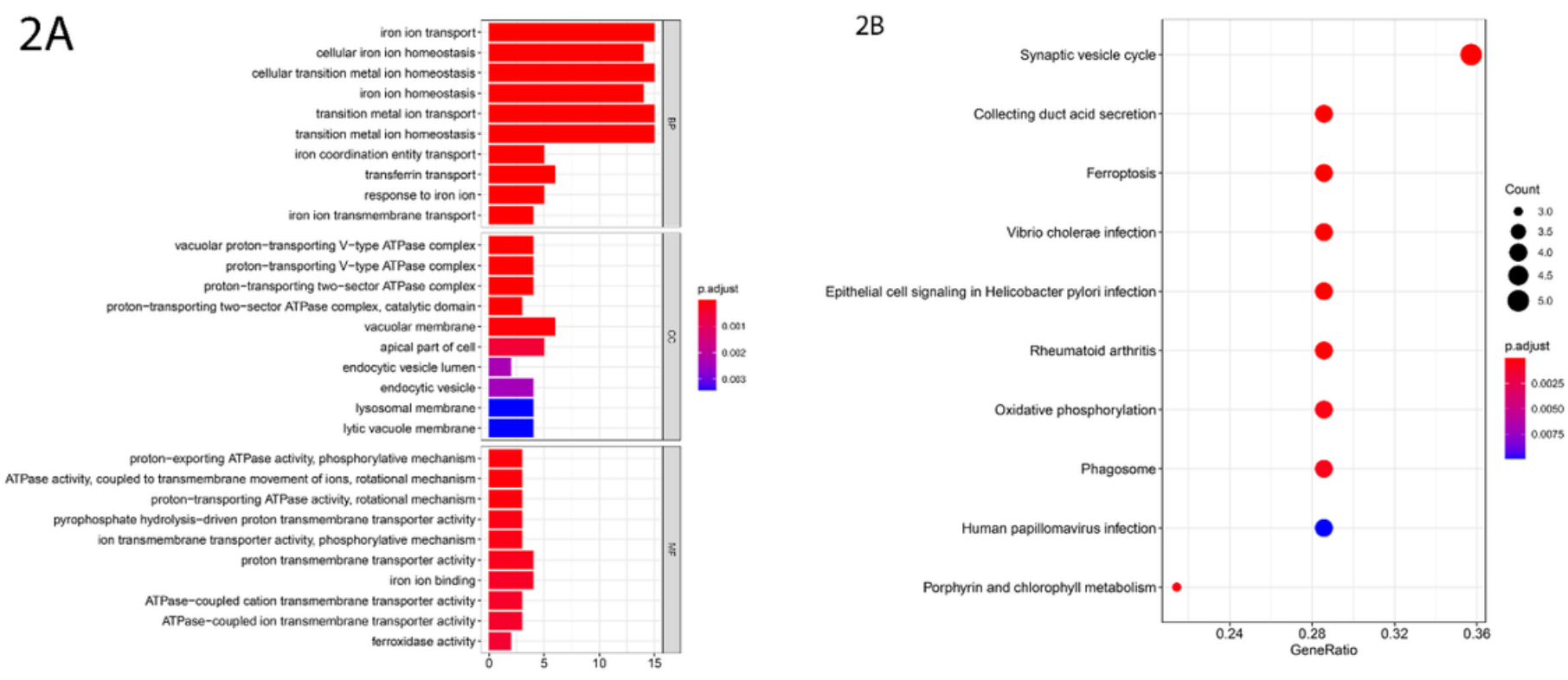

Figure 2

GO an KEGG functional enrichment analysis of DEIMRGs A) GO functional annotation of DEIMRGs in HCC B) KEGG pathway of DEIMRGs in HCC

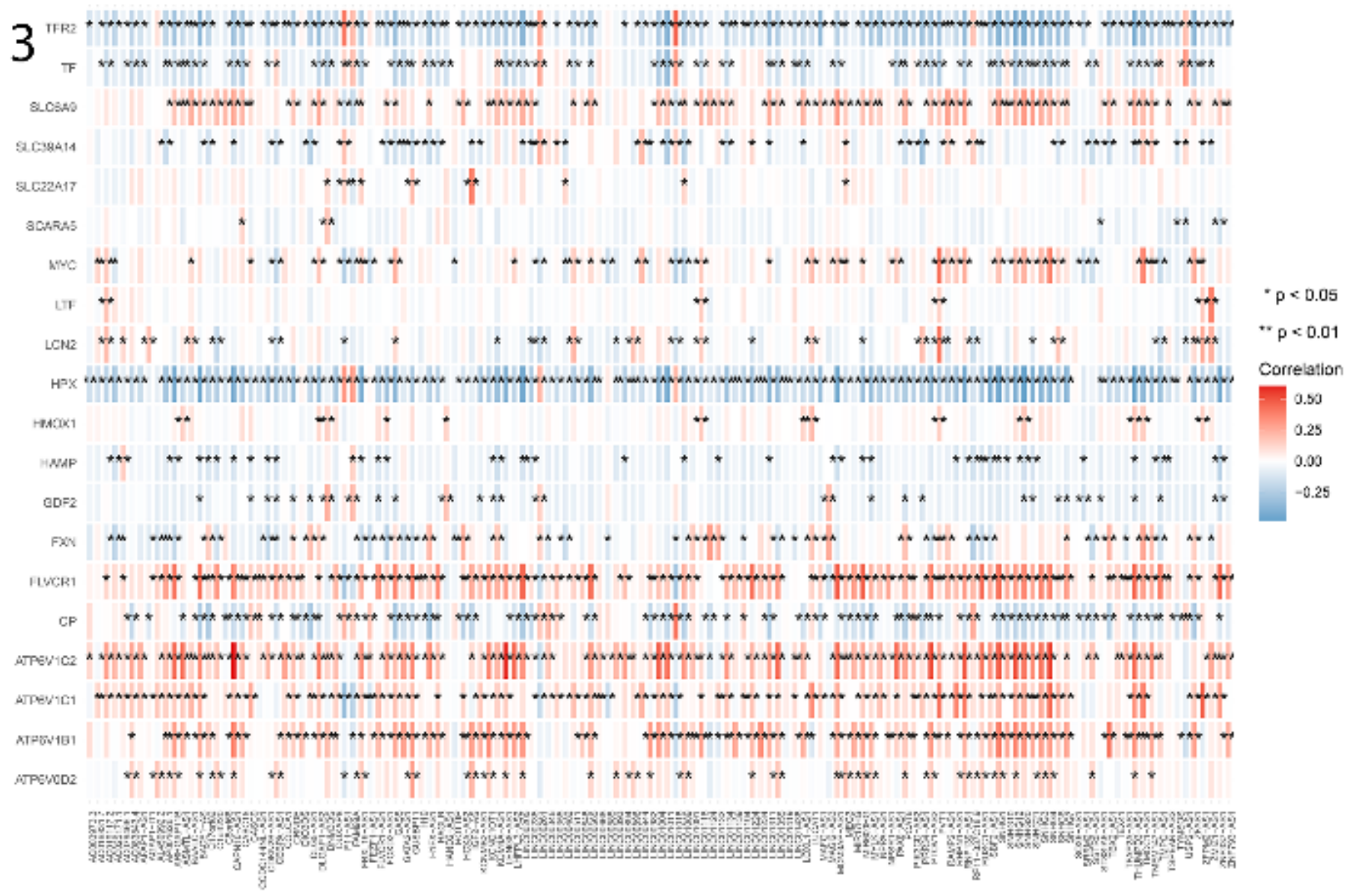

Figure 3 
Identification of the iron metabolism-related DElncRNAs by pearson correlation analysis
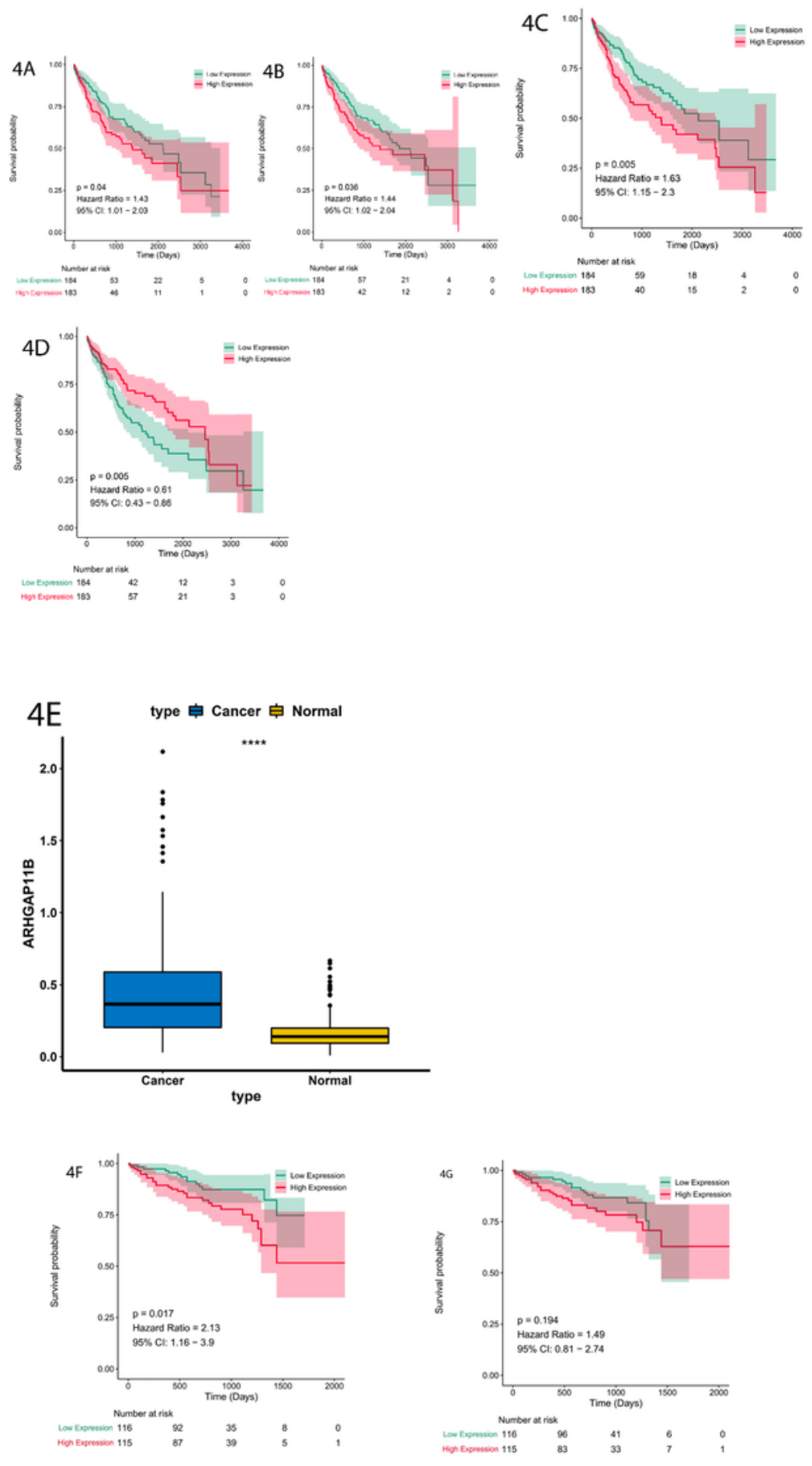

\section{Figure 4}

Kaplan-Meier survival curves of iron metabolism-related DElncRNAs from the TCGA cohort in HCC and validate the survival results in ICGC portal in ICGC AD Kaplan-Meier survival curves of ARHGAP11B from the TCGA cohort B囚 Kaplan-Meier survival curves of LINC00205 from the TCGA cohort CQ Kaplan-Meier survival curves of SNHG12 from the TCGA cohort DQ Kaplan-Meier survival curves of LINC00261 from the 
TCGA cohort EX Expression analysis of ARHGAP11B using ICGC data F囚 Kaplan-Meier survival curves of ARHGAP11B using ICGC data G $\triangle$ Kaplan-Meier survival curves of SNHG12 using ICGC data The red lines represented the high expression of iron metabolism-related DElncRNAs in HCC,and the green lines represented low expression of iron metabolism-related DElncRNAs
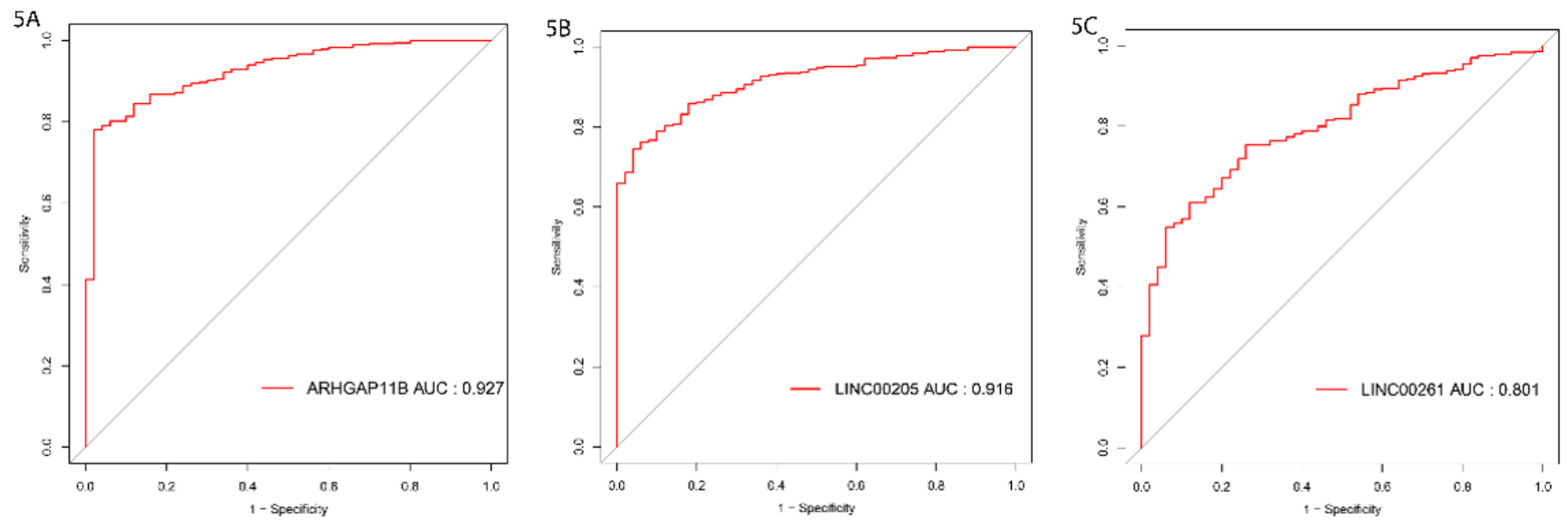

Figure 5

ROC curve analysis of three iron metabolism-related DElncRNAs in TCGA cohort in HCC A) ROC curve analysis of ARHGAP11B in the TCGA B) ROC curve analysis of LINC00205 in the TCGA C) ROC curve analysis of LINC00261 in the TCGA The X-axis represented the total survival time (year) and the Y-axis represented the survival rate. 

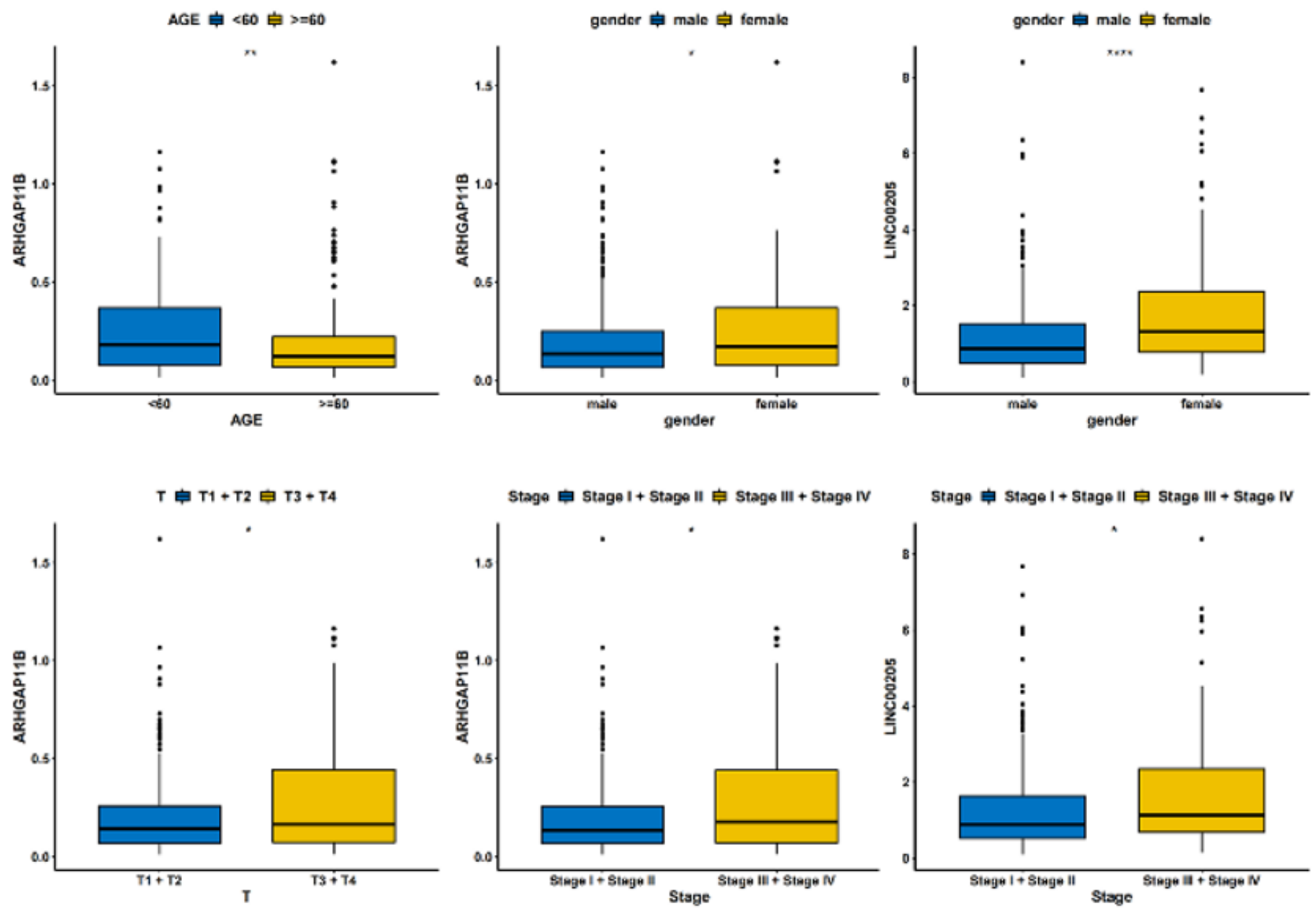

Figure 6

the expression of the possible prognostic DElncRNAs in different subgroups according to different clinicopathologic feature

$\begin{array}{llr}\text { 7A } & \text { pvalue } & \text { Hazard ratio } \\ \text { LINC00205 } & <0.001 & 1.003(1.001-1.004) \\ \text { LINC00261 } & 0.325 & 1.000(1.000-1.000) \\ \text { ARHGAP11B } & 0.003 & 1.002(1.001-1.003) \\ \text { Age } & 0.205 & 1.273(0.877-1.847) \\ \text { Gender } & 0.205 & 0.783(0.537-1.143) \\ \text { M } & 0.229 & 0.771(0.505-1.178) \\ \text { N } & 0.353 & 0.822(0.543-1.243) \\ \text { T } & <0.001 & 1.644(1.353-1.999) \\ \text { Stage } & <0.001 & 1.665(1.356-2.044)\end{array}$
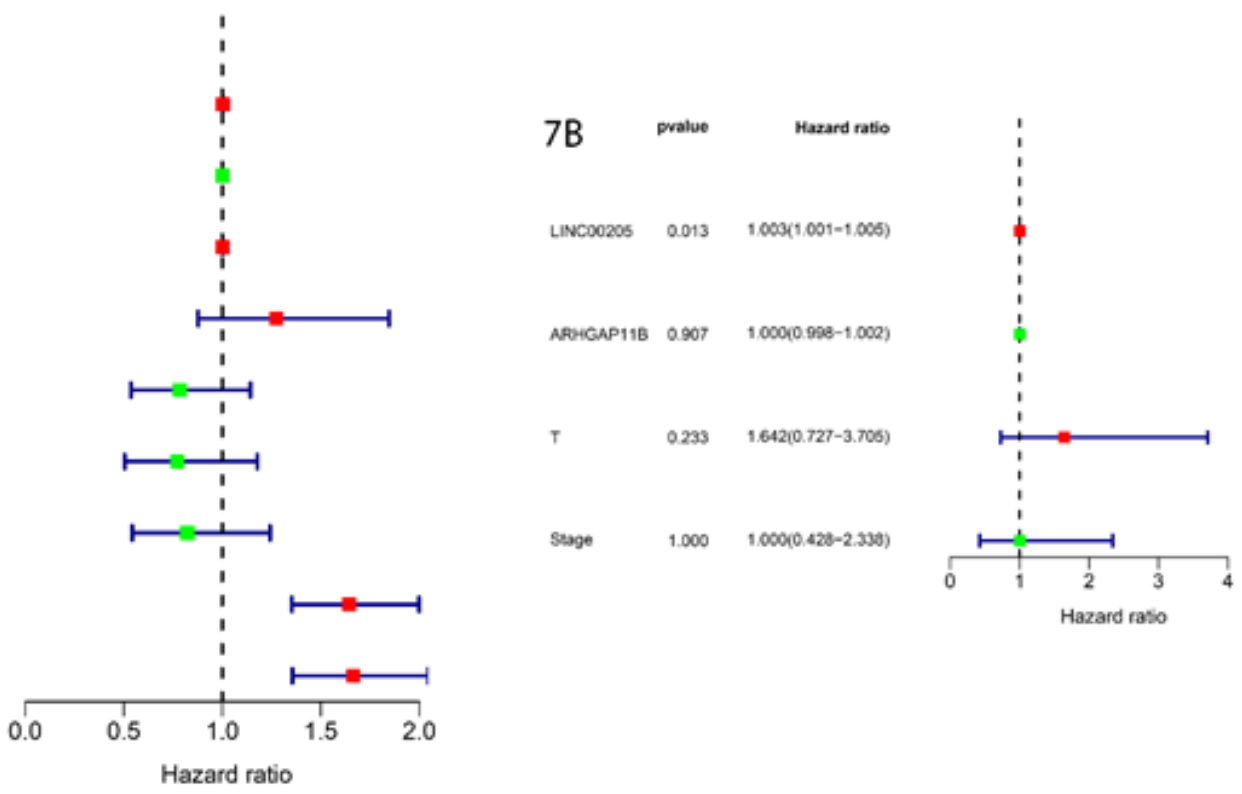
Figure 7

univariate and multivariate Cox regression analysis of iron metabolism-related DElncRNAs and clinicopathological parameters A) univariate Cox regression analysis of iron metabolism-related DElncRNAs and clinicopathological parameters B)multivariate Cox regression analysis of iron metabolism-related DElncRNAs and clinicopathological parameters

$8 \mathrm{~A}$

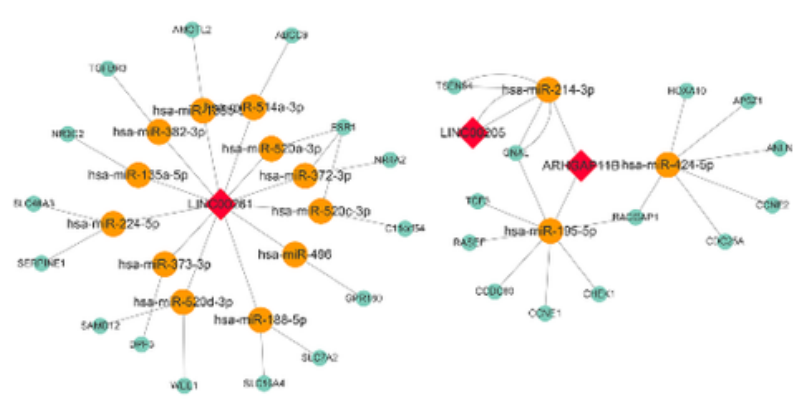

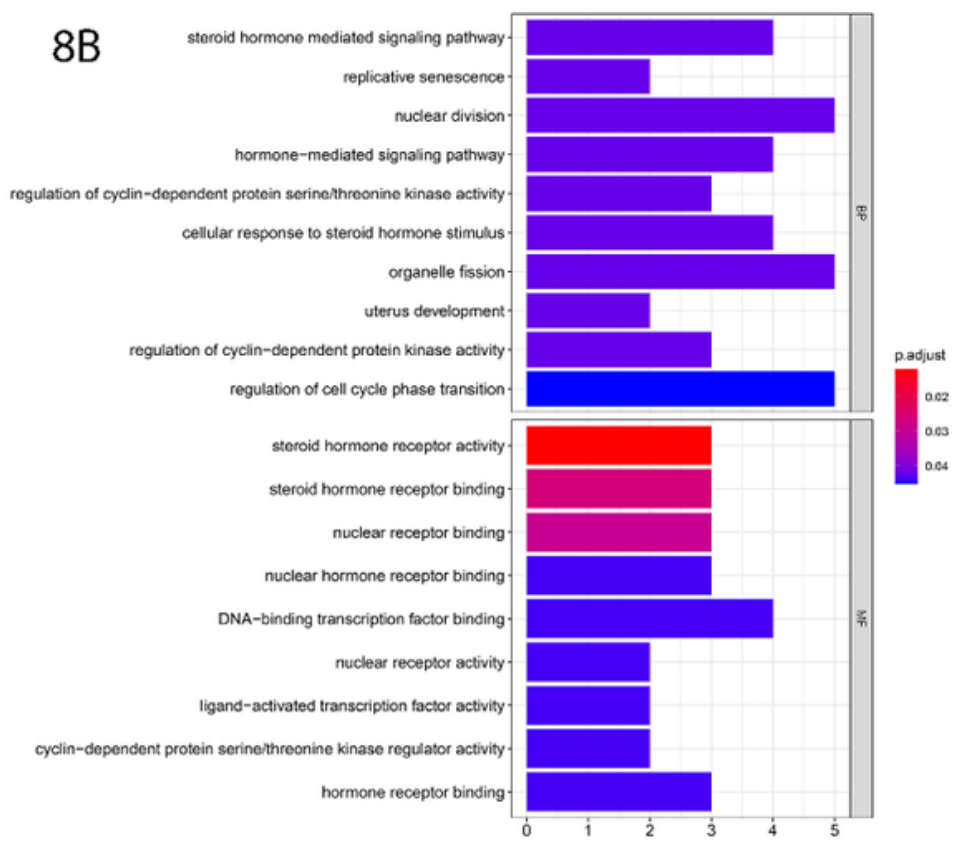

8D
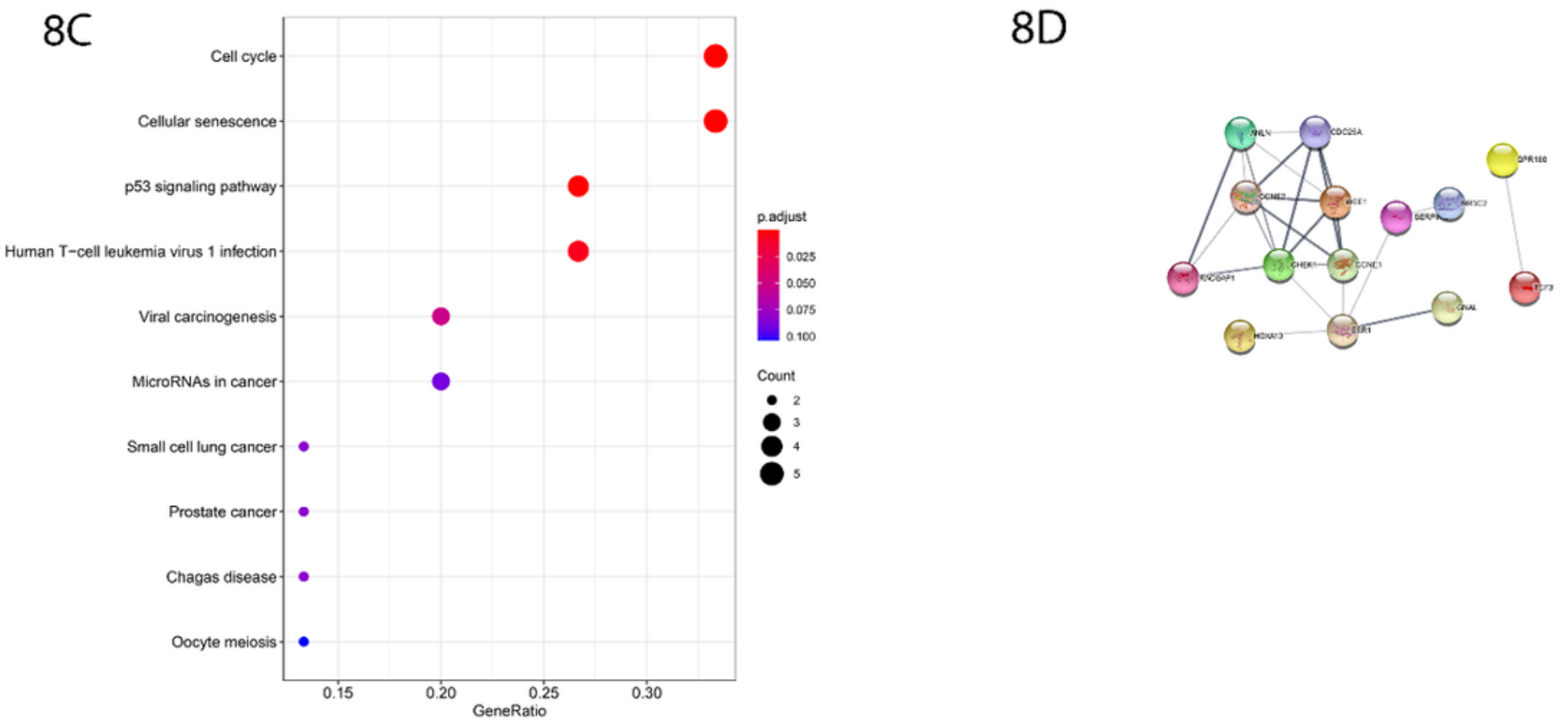

Figure 8 
ceRNA regulatory network based on iron metabolism-related DElncRNAs and functional analysis A) Construction of the ceRNA regulatory network B) GO function analysis of the genes in the constructed ceRNA regulatory network C) KEGG pathway function analysis of the genes in the constructed ceRNA regulatory network $D$ ) protein-protein interaction networks of the genes in the constructed ceRNA regulatory network 